This item was submitted to Loughborough's Research Repository by the author.

Items in Figshare are protected by copyright, with all rights reserved, unless otherwise indicated.

\title{
An investigation of three-dimensional scanning of human body surfaces and its use in the design and manufacture of prostheses
}

PLEASE CITE THE PUBLISHED VERSION

PUBLISHER

Professional Engineering Publishing / @ IMechE

VERSION

VoR (Version of Record)

LICENCE

CC BY-NC-ND 4.0

\section{REPOSITORY RECORD}

Bibb, Richard J., P. Freeman, R. Brown, Adrian Sugar, Peter L. Evans, and Alan Bocca. 2019. "An Investigation of Three-dimensional Scanning of Human Body Surfaces and Its Use in the Design and Manufacture of Prostheses". figshare. https://hdl.handle.net/2134/5711. 
This item was submitted to Loughborough's Institutional Repository (https://dspace.lboro.ac.uk/) by the author and is made available under the following Creative Commons Licence conditions.

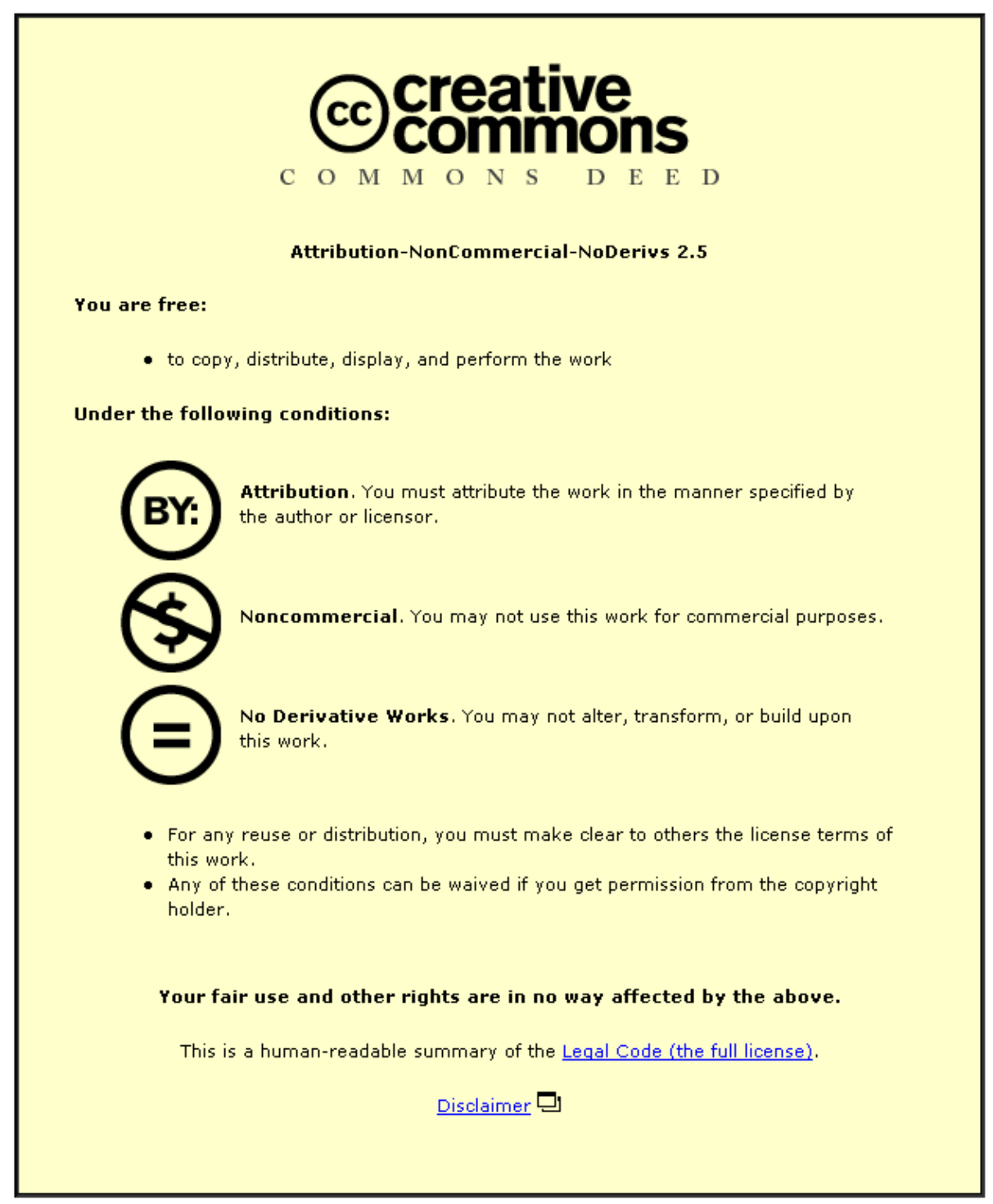

For the full text of this licence, please go to: http://creativecommons.org/licenses/by-nc-nd/2.5/ 


\title{
An investigation of three-dimensional scanning of human body surfaces and its use in the design and manufacture of prostheses
}

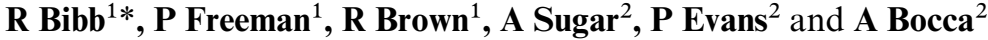 \\ ${ }^{1}$ Design Engineering Research Centre, University of Wales Institute, Cardiff, Wales, UK \\ ${ }^{2}$ Maxillofacial Unit, Morriston Hospital, Swansea NHS Trust, Swansea, Wales, UK
}

\begin{abstract}
The capture of highly accurate data describing the complex surfaces of the human body may prove extremely useful in many medical situations. The data provide a method of measuring and recording changes to the surface of a patient's soft tissue. The data may be applied to computercontrolled manufacturing techniques, such as rapid prototyping (RP). This enables accurate physical replicas of the patient topography to be produced. Such models may be used as an aid in the design and manufacture of prostheses.

This paper describes an investigation aimed at identifying problems that may be encountered when scanning patients and describes the application of the resulting data in the design and manufacture of facial prostheses. The results of the experiment are presented together with a discussion of the accuracy and potential advantages afforded by this approach.
\end{abstract}

Keywords: three-dimensional scanning, rapid prototyping, facial prostheses, prosthesis manufacture

\section{INTRODUCTION}

Three-dimensional surface scanning, or reverse engineering, has been used in industry for many years as a method of integrating the surfaces of complex forms with computer-generated design data [1]. Non-contact scanners operate by using light and camera technology to capture the exact position in space of points on the surface of objects. Computer software is then used to create surfaces from these points. These surfaces can then be analysed in their own right or integrated with computer aided design (CAD) models. The scanner used in the work described in this paper is an optical system that uses a fringe pattern of white light and digital camera technology to capture approximately 140000 points on the surface of an object (Steinbichler USA, 40000 Grand River, Novi, Michigan). Scanners using this type of moire fringe pattern have been used in the past in the assessment of spinal deformity [2]. In this case the area to be scanned is distinguished from its surroundings by altering the contrast. For example, a white object may be placed on a dark background and vice versa.

The MS was received on 19 November 1999 and was accepted after revision for publication on 11 January 2000.

* Corresponding author: Design Engineering Research Centre, University of Wales Institute, Cardiff, Western Avenue, Cardiff CF5 2 YB, Wales, UK.
As the scanner depends on the observation of a projected fringe pattern on the object, problems are encountered when scanning transparent or highly reflective surfaces. These can be overcome by applying a fine powder that will give the object an opaque matt finish. The process also means that the scanner can only collect data on surfaces within the line of sight. Areas that are obscured or are at too great an angle to the line of sight will not appear in the scan data. This is overcome by moving either the object or the scanner and repeating the process. Depending on the shape of the object many scans may be necessary. The data from each of these scans are then aligned using software to give a single coherent data set.

Due to the very high accuracy of this type of scanner, nominally $0.05 \mathrm{~mm}$, movement of the object should be avoided during scanning. Even small movements will cause noise and affect the quality of the resulting data. When scanning human body parts the problem of eliminating movement of the subject becomes more apparent. For the best results, the patient may have to be braced in a comfortable position during each scan, in this case for approximately $40 \mathrm{~s}$. A similar system, being investigated for use in measuring and recording changes in a patient's topography, uses multiple cameras and a fast capture time to eliminate the problems associated with motion. However, a smaller number of data points are captured at 
a slightly lower accuracy [3]. Other systems based on scanning have been used to manufacture customized orthotics for podiatric patients $[\mathbf{4 , 5}$ ]. To date the application of captured surface data in the manufacture of facial prostheses has not been fully investigated.

The human face offers many potential applications that could exploit this technology. However, scanning human faces presents particular problems. A primary difficulty is presented by the presence of hair. Hair does not form a coherent surface and the scanner will not pick up data from areas such as the eyebrows and lashes. This problem can be overcome to a certain degree by dusting a fine white powder over the hair. When considering the scanning of faces the area around the eyes may also be particularly difficult. As described above, movement leads to the capture of inaccurate data, to minimize problems caused by blinking during the scan it is more comfortable for the subject's eyes to be kept closed. This also alleviates the discomfort caused by the bright light emitted by the scanner. If the eye is held open during the scan watering of the eye may cause problems. In addition, the surface of the eyeball is highly reflective making data capture difficult.

Line of sight issues are also encountered when scanning faces. For example, a single scan will not acquire data where the nose casts a shadow (see Fig. 1). However, this is overcome by taking several overlapping scans.

This paper describes how these issues were approached by an investigation into the scanning of human faces. The paper goes on to describe the application of these techniques in the manufacture of a prosthesis to restore the appearance of a patient recovering from the excision of a rare form of tumour.

\section{METHODS}

\subsection{Preliminary trial of facial scanning}

As a preliminary investigation of the practicality of scanning human faces, a male subject was scanned using the

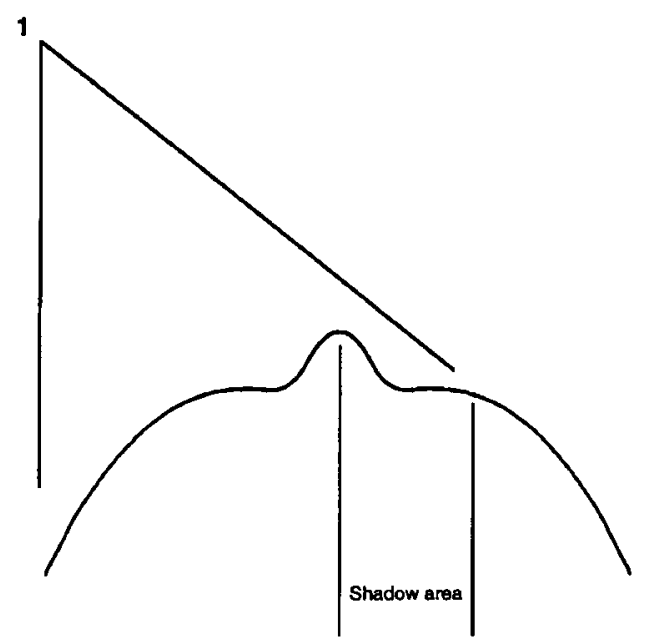

system described above. Initial attempts at scanning the face of the seated subject were poor due to slight involuntary movement of the head. Therefore, a support was fashioned from a block of polystyrene foam to locate the back of the head and minimize movement. With the subject thus supported in a semi-reclining position a series of three scans were taken, each from a different view point, similar to those shown in Fig. 1. A fourth scan was taken from a position below the second scan to allow the acquisition of data from the area below the eyebrow ridge. Each scan took approximately $40 \mathrm{~s}$, during which the subject must remain motionless. The whole process of arranging the subject and taking these four scans took approximately $10 \mathrm{~min}$.

Once completed, the scans were aligned using proprietary scanner software. To achieve this alignment, four notable points or landmarks were manually selected in an overlapping area in each of two separate scans. The software then aligned the landmarks and calculated the best fit between the two data sets. Consequent scans were aligned in a similar fashion.

In this experiment, four scans resulted in the capture of accurate data describing the whole face with the exception of areas obscured by hair, such as eyebrows, eyelashes and facial hair. These areas can be clearly seen as black in Fig. 2. Other areas lacking data, not immediately obvious in the figure, include areas beyond the line of sight, such as the nostrils. It was therefore concluded that the approach could be applied to the scanning of patients provided that the patients could be kept still during scanning.

\subsection{Scanning a surgical subject}

In this case, the subject was a patient recovering from reconstructive craniofacial surgery. The surgery required to excise a tumour had necessitated the removal of bone and soft tissue including the left eye. After successful operations to replace the orbital rim, an osseo-integrated

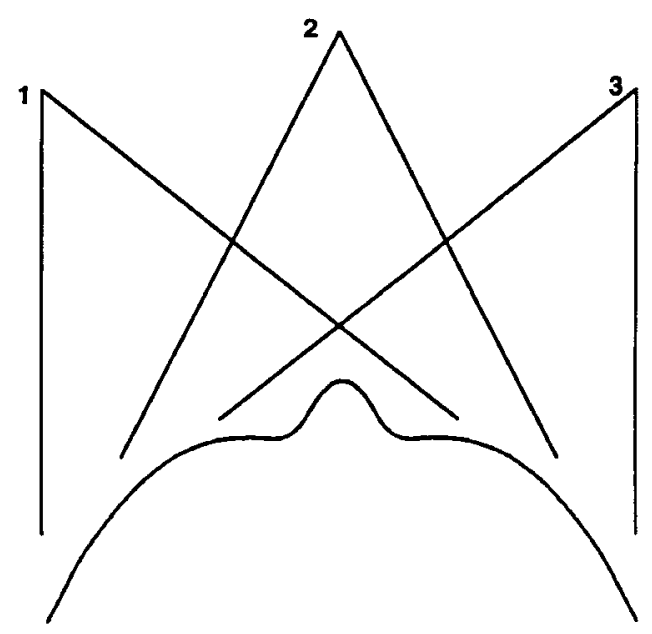

Fig. 1 Line of sight and view points for scanning the face 


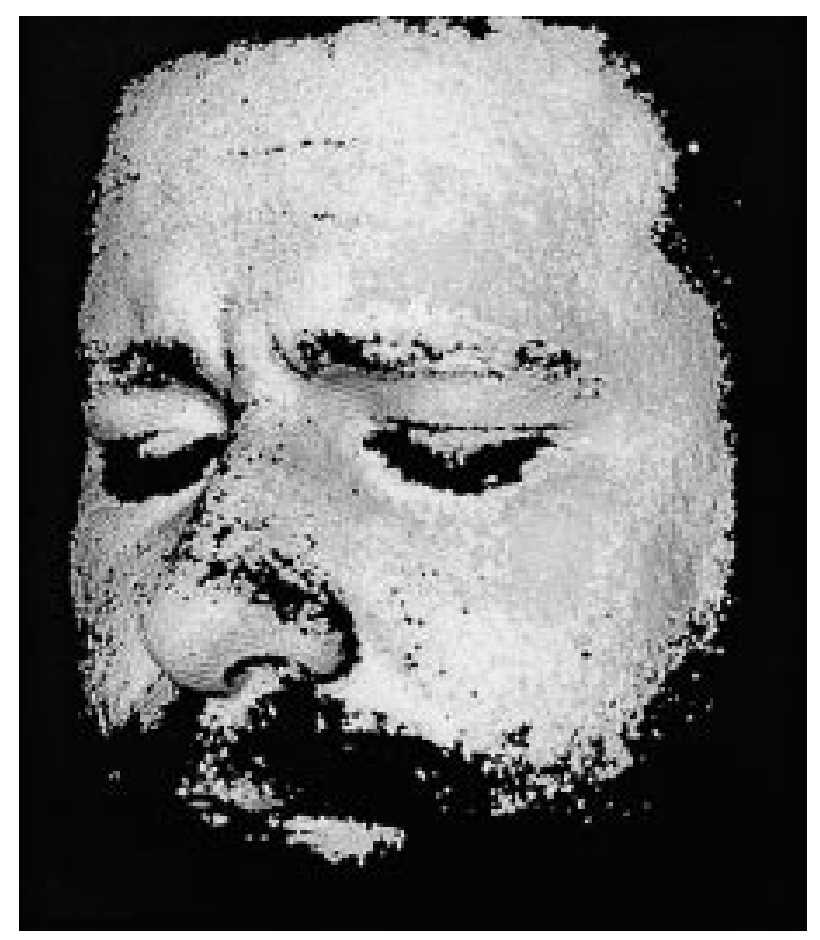

Fig. 2 Scan data of the face of a male subject

(bone anchored) prosthetic was planned for the missing eye and surrounding tissue [6]. To aid in the construction of this prosthesis the right (unaffected) side of the patient was scanned and the data were used to create a laterally inverted ('mirrored') model that would be used as a guide when creating the prosthesis.

Four scans were taken of the patient's face with the chin supported on a polystyrene block to minimize movement. As the data were intended to aid in the construction of a prosthetic of an open eye, a scan of the open eye was attempted. The scans were taken from angles similar to those shown previously, however, care was taken to ensure that the bright light from the scanner did not shine directly into the patient's eye. As before, data were not captured from areas obscured by the eyebrows and lashes. Small unavoidable movements of the eye and eyelids and the reflective nature of the surface of the eye itself affected the accuracy of the captured data. However, as this inaccuracy was extremely small it did not affect the overall quality of the data (see Fig. 3). As with the previous subject, the scanning of the patient took approximately $10 \mathrm{~min}$.

The next step required the building of a model of the area around the unaffected eye. To further aid the creation of the prosthesis the data would be laterally inverted ('mirrored' left to right) before building the model. This model could then be used to guide the production of a prosthetic with good size, fit and aesthetic symmetry.

To create a model from the scan data, the data were translated into an STL file format [7]. This is a triangular facetted surface normally used in rapid prototyping systems (see Fig. 4). However, before the data can be used by such systems to produce a model, any gaps in the data, such as the area at the eyebrows, have to be filled. This is achieved by using surface creation software to create a patch that continues the shape of the captured data surface. The patch was created to follow the natural curves of the surrounding data and replicate the surface as best as possible. This required a certain amount of judgement on the part of the operator. However, this

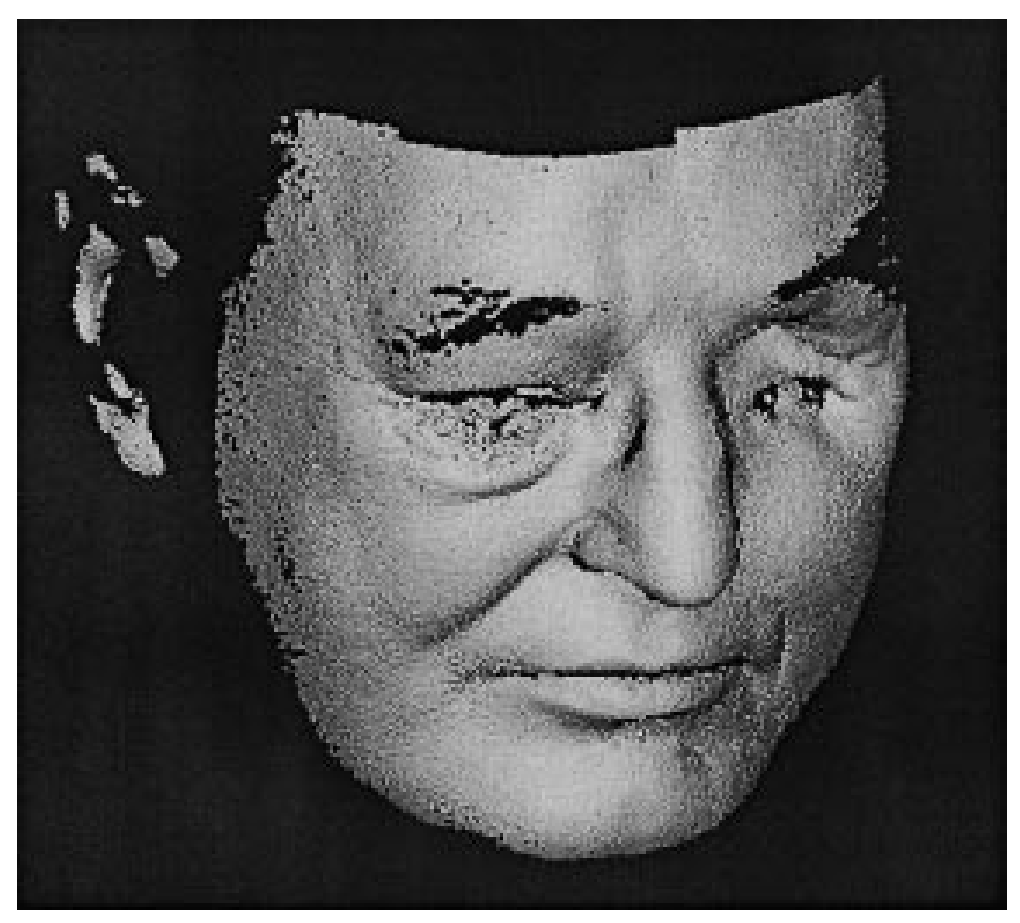

Fig. 3 The aligned scan data 


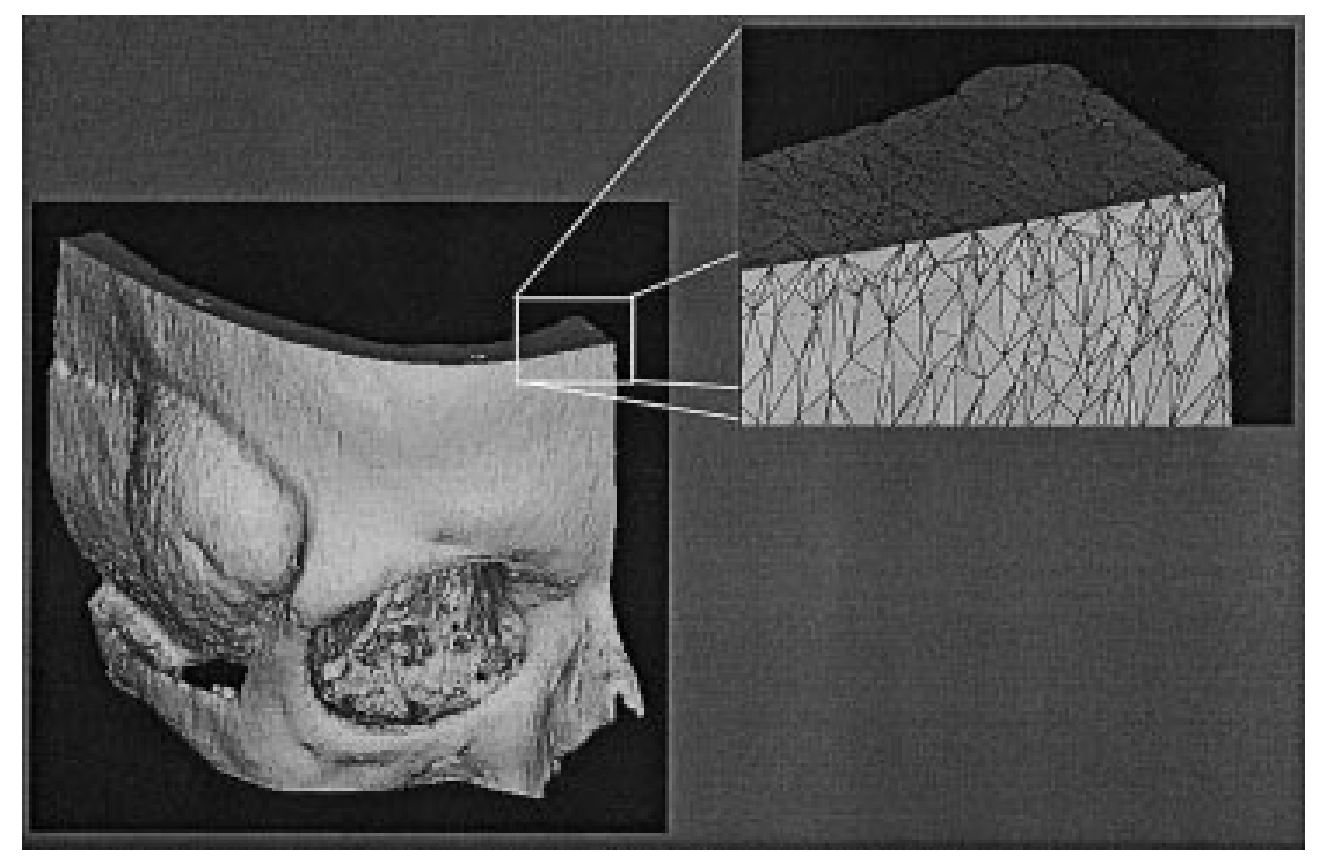

Fig. 4 Close-up of an STL file

case did not present great difficulty in this respect, as the missing areas were relatively small.

The file size can be reduced at this stage by removing unnecessary points. This can be achieved without sacrificing accuracy because there are vast numbers of points in the captured data coupled with the fact that the accuracy of the scan data is greater than can be achieved by subsequent rapid prototyping processes. An offset surface was created from the captured data and the gap between them closed to create a finite bound volume. To minimize file size and model cost only the specific area of interest was selected. The resulting data were stored as an STL file. The STL file size was reduced to 3.6 Mbytes. This was then laterally inverted ('mirrored') as shown in Fig. 5. In this case, the process of creating a valid STL file from the scan data took approximately $3 \mathrm{~h}$, but this will vary from case to case.

The STL file format is considered a de facto standard in rapid prototyping and may therefore be used in any number of rapid prototyping processes, such as stereolithography, laminated object manufacture, selective laser sintering or fused deposition modelling [8]. The model in this case was produced by the Design Engineering Research Centre using laminated object manufacturing (LOM) (see Fig. 6). The data may be archived for future reference or used to reproduce the model if required.
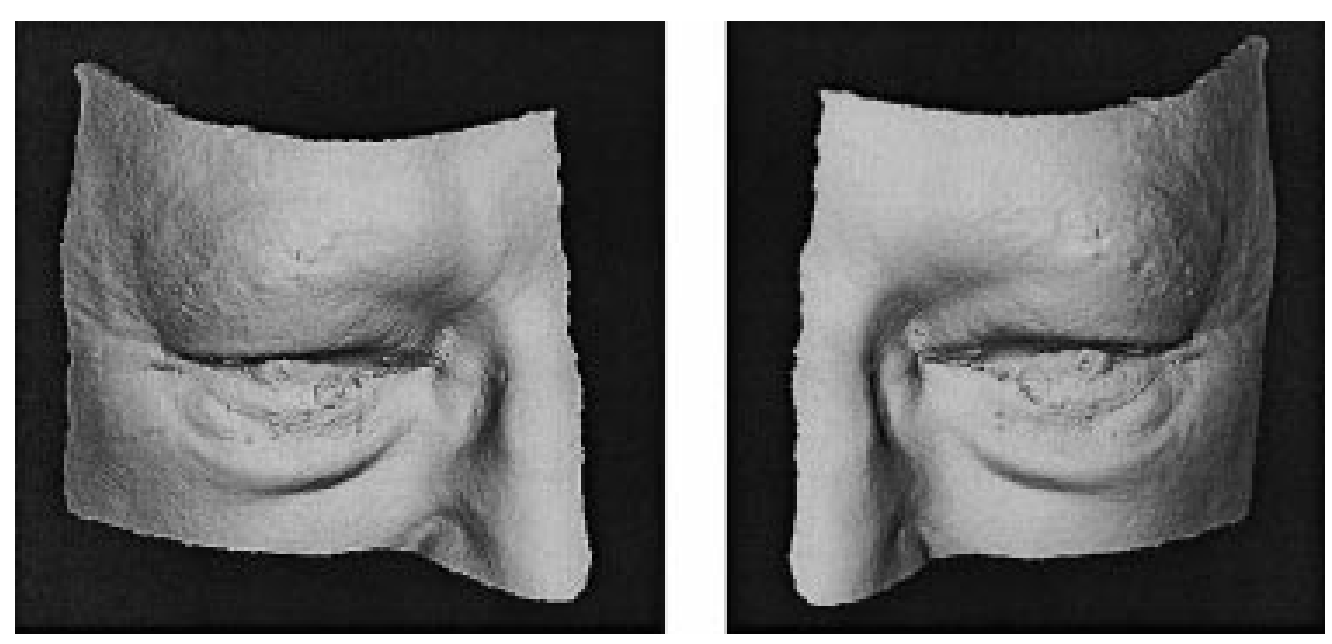

Fig. 5 STL file and 'mirrored' file of the unaffected eye 


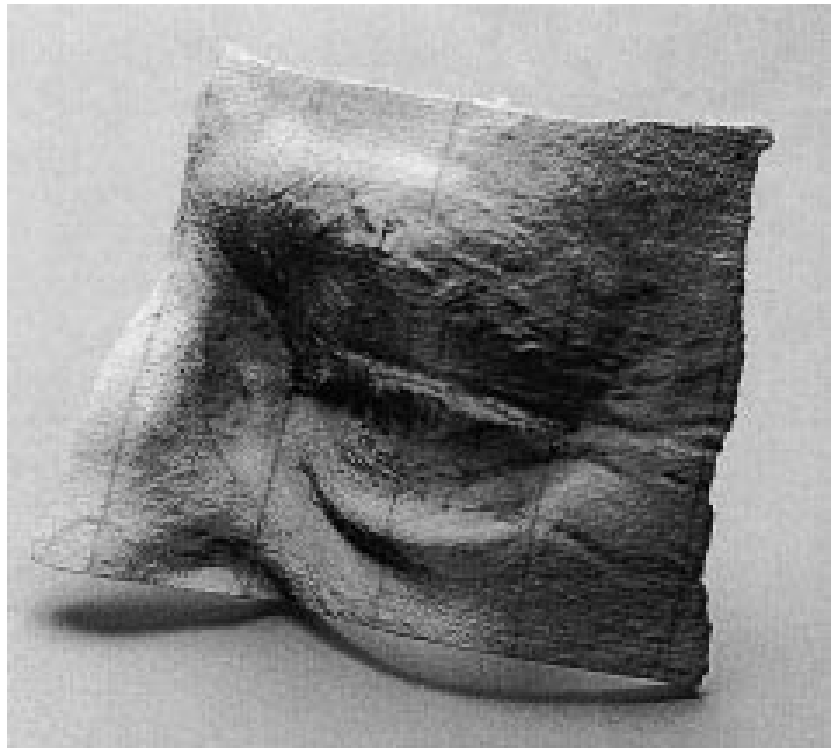

Fig. 6 An LOM model of the mirrored unaffected eye

\subsection{Prosthesis manufacture}

This case involved the manufacture of an osseointegrated silicone prosthetic of the left orbit. Previously two titanium fixtures had been attached to the zygomatic bone and allowed to integrate to the bone. Six months later they were exposed and per-cutaneous titanium abutments attached to them. The eventual prosthesis would attach to these abutments with magnets. To aid the construction of the prosthesis the LOM model was used to cast a wax replica. The prosthetist then removed excess material from the wax until it approximated the required shape. The traditional procedure would have required the prosthetist to carve this piece from wax. This would have taken the prosthetist approximately half a day in this particular case, during which the patient would have been required to sit with the prosthetist for visual reference. The use of the model therefore, not only saved approximately half a day of work for the prosthetist (from a total of 3) but importantly, also reduced the time required for the patient to attend the clinic.

The aperture for the eye was opened to allow the positioning of the artificial eye. This is considered crucial to the overall success of the prosthesis [6]. It was noted that, compared with the traditional methods, the mirrored nature of the model allowed far greater accuracy when locating the artificial eye, especially concerning anterior-posterior positioning. Once the eye position was fixed, the fine details were built up in wax. The areas immediately around the eye were dealt with in particular as this is where the original scan data, and therefore the LOM model, had lost some detail.

An impression was taken from the patient and used to shape the rear surface of the prosthesis. A small acrylic base plate that would hold the magnets used to locate the prosthesis was also made. When the prosthetist was satisfied with the visual appearance of the fine details and the fit of the prosthesis it was cast in colour-matched silicone in the usual manner.

\section{RESULTS}

\subsection{Accuracy}

The accuracy of the scan data is nominally $0.05 \mathrm{~mm}$. From the data, the theoretical height of the model was $76.76 \mathrm{~mm}$. LOM models are nominally accurate to within $0.2 \mathrm{~mm}$. However, when measured, the height of the completed LOM model was found to measure $76.7 \mathrm{~mm}$. Therefore, the accuracy of the model can be estimated to be in the order of $\pm 0.1 \mathrm{~mm}$. As all human faces are somewhat asymmetric and the surface of the skin is somewhat pliable, the wax replica was manually manipulated and adjusted to fit the desired area. Therefore, an accuracy of around $0.1 \mathrm{~mm}$ is more than adequate for prosthesis manufacture. The model also proved to be a good match in terms of reproducing a realistic visual appearance for the prosthesis.

\subsection{Outcome analysis}

The success of this experiment proves the feasibility of three-dimensional scanning of human body surfaces. The ease and relative speed of the scanning allow the complex forms of human features to be permanently captured without hindrance or discomfort to the patient. The accuracy of the data was found to be more than adequate for prosthesis construction. This method would compare favourably with the current practice of taking impressions, proving to be quicker, more accurate and aiding the reproduction of a realistic visual appearance. In particular, the use of 'mirrored' medical models was felt to be of great help to the prosthetist when positioning artificial eyes in orbital prostheses.

The cost of the scanning equipment is considerable and it may be difficult for hospitals to justify the initial investment. For this reason, it may prove more feasible for hospitals to use external service providers for the cases where this approach is expected to produce superior results. The cost of the scanning described in this paper would probably amount to several hundred pounds, with the LOM model costing approximately $£ 120$. The costs incurred by this approach should be balanced against the improved results and crucially the time saved over traditional methods. The reduction in time taken allows more patients to be treated, thereby reducing waiting lists (a major goal of the British National Health Service).

The non-contact nature of the scanning means there is less discomfort for the patient and no distortion of soft tissues caused by the pressure applied when taking 
impressions. This advantage in combination with the ability to 'mirror' data may have many applications in rehabilitation. It is difficult, for example, to take a satisfactory impression of a breast, therefore a similar technique may be used in the creation of symmetrical prostheses for mastectomy patients. However, care should be taken when scanning the body surface to ensure it is in the position that relates to the intended use. For example, body parts that are weight bearing will distort according to the position and posture of the patient. From the results of this case study, it can be concluded that three-dimensional scanning and medical modelling can save a significant amount of time for both the patient and the prosthetist. Lateral inversion and high accuracy can be a significant aid in prosthesis manufacture, especially for large or complex cases. These techniques may be a valuable aid to shaping and positioning the prosthesis but the skill and knowledge of the clinicians will determine the best method of creating, colour matching and attaching the prosthesis to the patient.

\section{ACKNOWLEDGEMENTS}

The authors would like to thank the patient for his cooperation in this investigation. The authors gratefully acknowledge the work of Terry Cross and Tim Williamson of the Design Engineering Research Centre (DERC), who respectively carried out the scanning and conducted the data manipulation and STL file generation, and Paul Bateman, also of the DERC, who produced the LOM model.

\section{REFERENCES}

1 Motavalli, S. Review of reverse engineering approaches. Computers Ind. Engng, 1998, 35(1-2), 25-28.

2 Wong, H. K., Balasubramaniam, P., Rajan, U. and Chang, S. Y. Direct spinal curvature digitization in scoliosis screening: a comparative study with moiré contourography. J. Spinal Disorders, 1997, 10(3), 185-192.

3 Tricorder Technology plc. Tricorder ships new measurement software. Press Release, 8 December 1998, Tricorder Technology Limited, Harefield, Middlesex.

4 Bergman, J. N. The Bergman foot scanner for automated orthotic fabrication. Clinics in Podiatry Medicine Surg. (Treatment Biomechanical Assessment Using Computers), 1993, 10(3), 363-375.

5 Bao, H. P., Soundar, P. and Yang, T. Integrated approach to design and manufacture of shoe lasts for orthopaedic use: reverse engineering in industry: research issues and applications. Computers Ind. Engng, 1994, 26(2), 411-421.

6 Branemark, P. and De Oliveira, M. F. (Eds) Craniofacial Prostheses, Anaplastology and Osseointegration, 1997, pp. 101-110 (Quintessence Publishing Company Inc., Carol Stream, Illinois).

7 Manners, C. R. STL File Format, 1993 (3D Systems Inc., Valencia, California).

8 Jacobs, P. F. Stereolithography and other RP\&M Technologies, 1996 (Society of Manufacturing Engineering, Dearborn, Michigan). 